\title{
Análise epidemiológica de pacientes com linfoma de hodgkin nos últimos cinco anos no estado do Rio de Janeiro
}

\author{
Epidemiological analysis of patients with hodgkin lymphoma in the last five years in the state of Rio \\ de Janeiro
}

Felipe Teixeira Freitas ${ }^{\dagger *}$, Juliana Lopes Dias ${ }^{\dagger}$, Bruno Cezario Costa Reis ${ }^{\ddagger}$

Como citar esse artigo. Freitas,

F.T.; Dias, J.L.; Reis, B.C. Análise epidemiológica de pacientes com linfoma de hodgkin nos últimos cinco anos no estado do Rio de Janeiro. Revista de Saúde. 2020 Jan./Jun.; 11 (1): 64-66.

\begin{abstract}
Resumo
O linfoma de Hodking constitui uma doença que resulta em uma neoplasia maligna que se dissemina por via linfática e afetando principalmente gânglios cervicais e o mediastino. Essa patologia tem como sua principal forma a linfocítica nodular e é um dos tipos mais curáveis, com radioterapia e quimioterapia. O presente estudo faz uma análise da epidemiologia dessa doença dando ênfase principalmente na faixa etária, número de óbitos e taxa de mortalidade relacionando-os ao perfil socioeconômico dos doentes em questão ao longo do período analisado. Sendo assim, os resultados tiveram como pontos principais as baixas incidência e taxa de mortalidade, além da conclusão de que se trata de uma doença complexa que precisa de um diagnóstico precoce visando o sucesso do tratamento com radioterapia e quimioterapia.

Palavras-chave: Linfoma, doença de hodgkin, epidemiologia.
\end{abstract}

\begin{abstract}
Hodking's lymphoma has caused a disease that results in a malignant neoplasm that spreads via the lymphatic route and mainly affects the neck and mediastinum. This pathology has as its main nodular lymphocytic form and is one of the most curable types, with radiotherapy and chemotherapy. The present study analyzes the epidemiology of this disease, emphasizing mainly the age group, number of deaths and mortality rates related to the socioeconomic profile of the injuries in question during the analyzed period. Thus, the results obtained as main points, such as low mortality rates and mortality rates, in addition to the conclusion that it treats a complex disease, which needs an early diagnosis, or the success of treatment with radiotherapy and chemotherapy.

Keywords: Lymphoma, hodgkin's disease, epidemiology.
\end{abstract}

Os Linfomas são neoplasias causadas por alterações em oncogenes ou genes supressores de tumor, que levam a um grande número de células tumorais do sistema imune e podem afetar tanto as células B quanto as T. O Linfoma de Hodgkin (LH) é caracterizado pela presença da célula de Reed Sternberg (CRS) e será o foco de nosso estudo ${ }^{1}$. O índice de mortalidade na Europa é 0,4 casos para 100.000 habitantes ao $\mathrm{ano}^{2}$. Já nos Estados Unidos, temos em torno de 8500 novos casos de LH anualmente, mas aproximadamente 1100 mortes anuais ${ }^{3}$. O LH possui um comportamento bimodal, com predominância na faixa etária entre 20 e 40 anos $\square$. Dividimos histologicamente em dois tipos: LH clássico ( $\mathrm{LHc}$ ) correspondendo $95 \%$ dos caso e LH de predominância linfocítica nodular (LHPLN) correspondendo $5 \%{ }^{2}$. Ainda distribuímos os LHc em subtipos sendo sua predominância: LHc esclerose nodular (LHCEN): 70\%; LHc celularidade mista (LHCCM): 20 a 25\%; HLc rico em linfócitos (LHCRL): 5\%; LHc com depleção de linfócitos (LHCDL): $<1$ por cento $^{3}$. O diagnóstico, de acordo com a Organização Mundial de Saúde (OMS), é realizado por biópsia do nódulo ou excisão de massa na procura de CRS. A imunofenotipagem difere entre o LHc e LHPLN, o primeiro positivo para CD15 e CD30 e ocasionalmente positivo para CD20 e o LHPLN com presença de CD20 e CD45 e negativo para CD15 e CD30². Apesar do aumento significativo no número de casos nos últimos 60 anos, é uma das neoplasias mais curáveis dentre os canceres com alta resposta terapêutica quando realizado com radioterapia e quimioterapia adequadas $\square$.

O Linfoma de Hodgkin é uma doença do sistema linfático, a qualde acordocom os resultados desse trabalho foi identificado que a mesma acomete, principalmente, 
a população ativa da sociedade. Ademais, a patologia não é de grupo social ou etnia, porque acomete todos os níveis da população, mostrando a grande importância de seu estudo. Outro fato importante se diz respeito ao avanço da medicina nos tratamentos, pois hoje, a doença apresenta um índice de mortalidade muito pequeno, porém apesar do incrível avanço, o LH ainda possui um grande gasto na saude pública, logo, faz de grande relevância um maior estudo no quesito da terapêutica, sobretudo na quimioterapia da qual é altamente eficaz. Por fim, o trabalho vem por meio de uma analise epidemiológica, como uma neoplasia apesar de curativa, apresenta grandes impactos.

\section{Objetivo}

Analisar o panorama do linfoma de Hodking no estado do Rio de Janeiro, nos últimos 5 anos, relacionando dados importantes como faixa etária, número de óbitos e taxa de mortalidade ao perfil socioecônomico dos pacientes acometidos, visando, assim, correlacionar a epidemiologia atual com os resultados obtidos.

\section{Materiais e Métodos}

Foi realizada uma revisão de literatura do

Tabela 1. Internações segundo faixa etária.

Lista Morb CID-10: Doença de Hodgkin

Período: Jan/2015 - Jan $/ 2010^{6}$

\begin{tabular}{ll}
\hline Faixa Etária & Internações \\
\hline TOTAL & $\mathbf{1 4 1 9}$ \\
\hline Menor de 1 ano & 2 \\
1 a 4 anos & 9 \\
5 a 9 anos & 58 \\
10 a 14 anos & 104 \\
15 a 19 anos & 170 \\
20 a 29 anos & 404 \\
30 a 39 anos & 242 \\
40 a 49 anos & 124 \\
50 a 59 anos & 161 \\
60 a 69 anos & 89 \\
70 a 79 anos & 46 \\
80 anos ou mais & 10 \\
\hline Fonte: Sistema de Informaçoes Hospitalares do SUS (SIH-SUS)
\end{tabular}

período de 2011 a 2019, sendo pesquisadas no Google Acadêmico e PubMed, tendo sido utilizadas as seguintes palavras para pesquisa: Linfoma; doença de hodgkin; epidemiologia. Associado a um levantamento de dados disponíveis no DATASUS - Sistema de Informações Hospitalares do SUS (SIH/SIS) do Ministério da Saúde sendo avaliado, no período de janeiro de 2015 a janeiro de 2020, as internações, ano de atendimento, taxa de mortalidade, óbitos, faixa etária, sexo, etnia e valor total.

\section{Resultados}

No estado do Rio de Janeiro, de acordo com o Datasus, foram internados 1419 pessoas com LH no período de janeiro de 2015 a janeiro de 2020 , com gasto total de R $\$ 2.616 .818,31$ e com taxa de mortalidade de 5,71 . Além disso, foi possível observar que a faixa etária mais afetada foi de 20 a 49 anos, com 770 pacientes, seguida da população de até 19 anos com $343 \mathrm{e}$, por último, a cima de 50 anos com 306 casos, conforme tabela 1. Quanto ao sexo, o masculino mostrou maior número de afetados com $52,5 \%$ do total, conforme tabela 2. Em relação a etnia, os brancos são os mais afetados com $37,2 \%$, seguido dos pardos com $33,4 \%$, sendo importante ressaltar que não há informação sobre a cor de 262 pacientes, ou seja, $18,46 \%$. Por fim, o número

Tabela 2. Internações segundo o sexo Lista Morb CID-10: Doença de Hodgkin Período: Jan/2015 - Jan/2010

\begin{tabular}{lc}
\hline Sexo & Internações \\
\hline TOTAL & $\mathbf{1 4 1 9}$ \\
\hline Masculino & 745 \\
Feminino & 674 \\
\hline \multicolumn{2}{c}{ Fonte: Sistema de Informações Hospitalares do SUS (SIH-SUS) }
\end{tabular}

de óbitos pela doença foi de 81 pacientes, tendo 2015 o maior número com 24 casos e desde então o número de óbitos vem diminuindo até 2019 com 10 no total $\square$ (tabela 3). Além disso outra questão evidenciada foi a econômica, pois existe um padrão de maior incidência do LH nos países em desenvolvimento aprendam um pico tanto em crianças quanto na segunda década de vida no qual se enquadra o Brasil $\square$. A infecção por HIV está associada com com o aumento do risco de neoplasias e dentre elas o LH $\square$. 
Tabela 3. Óbito segundo o ano de processamento

Lista Morb CID-10: Doença de Hodgkin

Período: Jan $/ 2015$ - Jan $/ 2010^{6}$

\begin{tabular}{ll}
\hline TOTAL & $\mathbf{8 1}$ \\
\hline 2015 & 24 \\
2016 & 15 \\
2017 & 14 \\
2018 & 15 \\
2019 & 10 \\
2020 & 3 \\
\hline
\end{tabular}

Fonte: Sistema de Informações Hospitalares do SUS (SIH-SUS) ${ }^{6}$

\section{Discussão}

A maioria dos pacientes irão apresentar uma linfadenopatia principalmente supradiafragmática, sendo que as formas ingnal e retroperitonial ocorrem em menor frequência. Cerca de um terço dos pacientes com LH apresentam sintomas inespecíficos como febre alta, perda de peso intensa, sudorese noturna e prurido crônico $\square$. Comoesperado, onúmerodecasos depacientes da faixa etária dos 20 a 49 anos foi a maior comparada as outras. Outro parâmetro de grande concordância com a literatura foi a taxa de mortalidade baixa, isso porque, com o avanço no tratamento, o número de óbitos pelo LH diminuiu consideravelmente $\square$. O valor gasto com o LH foi alto devido ao tratamento quimioterápico, que apesar de eficaz, ainda apresenta altos custos. Outro fato a ser evidenciado é que não existe uma grande discrepância em relação a etnia, porque o numero de casos entre negros e brancos foi semelhante $\square$.

\section{Conclusão}

O LH possui uma baixa incidência e taxa de mortalidade no estado do Rio de Janeiro, sendo que a população de maior incidência é a faixa etária dos 20 a 49 anos. Não há grandes diferenças no acometimento entre os sexos. Apesar dos custos com o tratamento da doença, a taxa de mortalidade é baixa quando tratada de forma eficaz, não representando um problema para a saúde pública. Diante disso, nota-se a complexidade do tema. Logo, para reduzir os números da mortalidade deve-se fazer diagnósticos precoces, pois quanto melhor for o estadiamento, melhor será o prognóstico do paciente. Assim, seria adequado fazer uma triagem em pacientes que estão na faixa etária de risco.

\section{Referências}

1. da-Silva F, Stroka A, Fusco V. ASPECTOS FARMACOLÓGICOS DO ANTICORPO MONOCLONAL (NIVOLUMAB) UTILIZANDO A VIA PD-1 NO TRATAMENTO ANTITUMORAL DO LINFOMA DE HODGKIN. Infarma - Ciências Farmacêuticas [Internet]. 2019 Apr 1; [Citado em: 2020 Mar 19]; 31(1): 13-19. Disponível em: http://revistas.cff. org.br/?journal=infarma\&page $=$ article \&op $=$ view $\&$ path $\% 5 \mathrm{~B} \% 5 \mathrm{D}=2294$

2. Eichenauer E, BMP A, M A, et al. Hodgkin lymphoma: ESMO Clinical Practice Guidelines for diagnosis, treatment and follow-up. [Internet]. 2018 [Citado em: 2020 Mar 25]. Disponível em: http://doi.org/10.1093/annonc/ mdy 080

3. L Siegel R, D Miller K, Jemal A. Cancer Statistics [Internet]. 2018 [Citado em: 2020 Mar 25]. Disponível em: https://doi.org/10.3322/caac.21442

4. Caroline Moreira da Silva L, da Costa Degiato T, Rezende S. M. de Oliveira B. LINFOMA DE HODGKIN: PREDOMINÂNCIA LINFOCÍTICA NODULAR, FISIOPATOLOGIA E DIAGNÓSTICO LABORATORIAL [Internet]. Biomedicina (2017); 2017 [Citado em: 2020 Mar 19]. Disponível em: http://www.unitoledo.br/repositorio/handle/7574/127

5. Bari A, Marcheselli R, Sacchi S. The classic prognostic factors in advanced Hodgkin's lymphoma patients are losing their meaning at the time of Petguided treatments. [Internet]. 2019 [citado em: 2020 Mar 18]. Disponível em: https://doi.org/10.1007/s00277-019-03893-7

6. DATASUS (SIH-SUS) - avaliado de jan de 2015 a jan de 2020 , avaliando internações, ano de atendimento, taxa de mortalidade, óbitos, faixa etária, sexo, etnia e valor total. Acesso em: 19/03/2020

7. Barros M, Hassan R, Niedobitek G. Disease patterns in pediatric classical Hodgkin lymphoma: a report from a developing area in Brazil. [Internet]. 2011 [citado em: 2020 Mar 26]. Disponível em: https://doi.org/10.1002/ hon. 984

8. Popova M. PARTICIPANT CLINICAL CASE DISCUSSION: NIVOLUMAB \& ASCT IN RELAPSED/REFRACTORY HIV-RELATED HODGKIN LYMPHOMA [Internet]. 2018 [citado em:2020 Mar 25]. Disponível em: https://oncologypro.esmo.org/meeting-resources/esmopreceptorship-on-lymphoma-lugano-november-2018/Participant-ClinicalCase-Discussion-Nivolumab-ASCT-in-Relapsed-Refractory-Hiv-RelatedHodgkin-Lymphoma

9. Ansell S. Hodgkin Lymphoma: Diagnosis and Treatment [Internet]. 2015 [citado em: 2020 Mar 18]. Disponível em: https://doi.org/10.1016/j. mayocp.2015.07.005 